\title{
Macrophage activation syndrome in adult systemic lupus erythematosus: report of seven adult cases from a single Italian rheumatology center
}

\author{
F. Dall'Ara', I. Cavazzana'2, M. Frassi ${ }^{2}$, M. Taraborelli ${ }^{2}$, M. Fredi², F. Franceschini' ${ }^{2}$, \\ L. Andreoli', M. Rossi ${ }^{3}$, C. Cattaneo ${ }^{4}$, A. Tincani ${ }^{1}$, P. Airò ${ }^{2}$ \\ ${ }^{1}$ Rheumatology and Clinical Immunology, Department of Clinical and Experimental Sciences, Spedali Civili \\ and University of Brescia, Italy; ${ }^{2}$ Rheumatology and Clinical Immunology, Spedali Civili of Brescia, Italy; \\ ${ }^{3}$ Internal Medicine, Spedali Civili of Brescia, Italy; ${ }^{4}$ Hematology, Spedali Civili, Brescia, Italy
}

Congresso: lavoro presentato al Congresso SIR, 2016.

\section{SUMMARY}

The aim was to describe the macrophage activation syndrome (MAS), a life-threatening syndrome characterized by excessive immune activation that can be triggered by conditions affecting immune homeostasis, in a cohort of adult Italian patients with systemic lupus erythematosus (SLE).

This was a monocentric retrospective evaluation. The utility of the H-score, developed to estimate the individual risk of having reactive MAS in adult patients, was assessed.

Among 511 patients with SLE, 7 cases (1.4\%) of MAS (all females) were identified and their medical records reviewed.

In all cases, MAS was simultaneous to the onset of SLE. All patients had fever, lymphadenopathy, hematological involvement, and high titer of anti-dsDNA antibodies. Workup for infections and malignancies was negative. In all cases, the H-score was higher than the cut-off suggested for the classification of reactive MAS. All cases required hospital admission, and 2 patients were admitted to the intensive care unit. Most patients were treated successfully with high doses of corticosteroids and with immunosuppressive drugs, whereas the full therapeutic regimen developed for primary hemophagocytic lymphohistiocytosis HLH was used only in one case. No death from MAS was observed.

MAS is a rare and severe disorder that complicated the onset of SLE in our cohort. The H-score may be useful in the classification of these patients.

Key words: Systemic lupus erythematosus; Macrophage activation syndrome; Adult; H-score.

Reumatismo, 2018; 70 (2): 100-105

\section{INTRODUCION}

$\mathrm{M}$ acrophage activation syndrome (MAS) is a severe and life-threatening disease, characterized by uncontrolled activation of the immune system with proliferation of $\mathrm{T}$ cells and well-differentiated non-neoplastic macrophages, which exhibit hemophagocytic activity (1). This massive systemic inflammatory response is associated with cytopenia, liver dysfunction, coagulopathy and extreme hyperferritinemia. It is classified in:

1) primary (or familial) hemophagocytic lymphohistiocytosis (HLH), a constel- lation of rare autosomal recessive disorders associated with various abnormalities in the cytolytic pathway;

2) secondary MAS, triggered by conditions affecting immune homeostasis, such as infections, malignancies, and autoimmune disorders (1).

MAS has been reported in association with almost any rheumatologic disorders, but the most common associations are, by far, with systemic juvenile idiopathic arthritis, adult-onset Still disease, and systemic lupus erythematosus (SLE) (1). MAS occurring in patients with SLE and other autoimmune diseases can be separated into two 
different entities: cases associated with an active infection, often as a complication of immunosuppressive therapy, and cases specifically associated with activity of the underlying disease (at its onset, or during a flare) (1).

Data on SLE-associated MAS are still limited. The first cases were described in 1991 as acute lupus hemophagocytic syndrome (2). Afterwards, several small case series were described, with an estimated prevalence of MAS among SLE patients ranging from $0.9 \%$ to $4.6 \%$ (3-8). To our knowledge, only one paper described adult SLE-associated MAS cases collected in Italy (9). However, in this study 9 SLE cases recruited from 2 rheumatology centers were presented together with 32 cases with other autoimmune diseases, and SLE cases were not separately analyzed (9).

Diagnostic criteria for MAS secondary to adult autoimmune diseases suffer from substantial limitations. The recognition that MAS is clinically similar to HLH led some authors to recommend the use of the HLH-2004 diagnostic guidelines; however these were developed primarily for homozygous genetic disorders leading to familial HLH (10), and have never been validated in adults or in the reactive form of MAS. Moreover, some of the proposed criteria (e.g., natural killer cell activity, soluble interleukin-2 receptor level) are not measured in routine practice. Recently, preliminary diagnostic guidelines for MAS complicating systemic juvenile idiopathic arthritis (11), or juvenile SLE (12), have been published, but these may not be suitable for adult patients. Finally, a diagnostic score (H-score) was developed with the aim of estimating the individual risk of having MAS for adult patients (13). However, the majority of patients in this study population had haematological malignancy and/or infection, and only $3.5 \%$ of them had SLE.

In the present study we describe the presentation and treatment of 7 adult SLE-associated MAS collected in a single Italian center. The possible utility of the $\mathrm{H}$-score was also evaluated.

\section{PATIENTS AND METHODS}

\section{Patients}

All adult patients with a diagnosis of SLE who were treated at the Rheumatology Unit of Spedali Civili, Brescia, Italy, between 1972 and 2014, were included in the present analysis. All patients satisfied both the 1997 revised criteria for the classification of SLE (14) and the 2012 Systemic Lupus International Collaborating Clinics (SLICC) criteria (15). Patients with a diagnosis of MAS formulated according to typical clinical and laboratory features and supported by clinicians' opinion were identified. All medical records were reviewed. In all cases the HLH-2004 classification criteria (10) were satisfied. Medical records were retrospectively reviewed using a standard data extraction form, evaluating: demographic data, disease duration, potential trigger factors for MAS onset, including, among other things, signs of active viral infections (this included, in all cases: Epstein Barr Virus, Cytomegalovirus, Hepatitis B Virus, Hepatitis C virus, B19 Parvovirus, Human Herpes Virus 8, Human Herpes Virus 6, Human Immunodeficiency Virus and adenovirus), as well as lupus clinical features, laboratory findings and treatment modalities for both SLE and MAS management. For each patient the SLE disease activity and the individual risk of having reactive MAS were evaluated using the SLE Disease Activity Index (SLEDAI-2K) score (16) and the H-score (13), respectively.

\section{Statistical analysis}

If not otherwise indicated, continuous variables were reported as median value and interquartile range (IQR).

\section{RESULTS}

Among the 511 patients with SLE followed at our center (age at diagnosis: $33 \pm 13 ; 92.2 \%$ female), seven $(1.4 \%)$ cases of MAS were identified (median age at diagnosis 31.5 years; IQR 26.5-42.5; all female). Their main clinical and laboratory features are reported in Table I. All 
Table I - Main clinical and laboratory features at diagnosis of SLE and MAS.

\begin{tabular}{|c|c|c|c|}
\hline \multicolumn{2}{|c|}{ Clinical features of MAS n (\%) } & \multicolumn{2}{|c|}{ SLE ACR classification criteria } \\
\hline $\begin{array}{l}\text { Fever } \\
\text { Haemorrhages } \\
\text { CNS dysfunction } \\
\text { Lymphadenopathy } \\
\text { Hepatomegaly } \\
\text { Splenomegaly }\end{array}$ & $\begin{array}{l}7(100 \%) \\
1(14 \%) \\
0(0 \%) \\
7(100 \%) \\
5(71 \%) \\
5(71 \%)\end{array}$ & $\begin{array}{l}\text { Cutaneous* }^{*} \\
\text { Arthritis } \\
\text { Nephritis } \\
\text { Serositis } \\
\text { CNS disease } \\
\text { Haematological involvement }\end{array}$ & $\begin{array}{c}5(71 \%) \\
3(43 \%) \\
0 \\
4(57 \%) \\
1(14 \%) \\
7(100 \%)\end{array}$ \\
\hline \multicolumn{2}{|c|}{ MAS laboratory parameters; median (IQR) } & \multicolumn{2}{|c|}{ Autoantibodies n (\%) } \\
\hline $\begin{array}{l}\text { WBC }\left(x 10^{3} / \mathrm{uL}\right) \\
\text { Neutrophils (x103/uL) } \\
\text { HGB (g/dL) } \\
\text { PLT (x103/uL) } \\
\text { AST (U/L) } \\
\text { ALT (U/L) } \\
\text { LDH (U/L) } \\
\text { Ferritin ( } \mu \mathrm{g} / \mathrm{L}) \\
\text { Fibrinogen (mg/dL) } \\
\text { Triglycerides (mg/dL) } \\
\text { ESR (mm) } \\
\text { CRP (mg/dL) }\end{array}$ & $\begin{array}{c}2.1(1.9-2.3) \\
1.3(0.9-1.8) \\
7.3(6.9-8.1) \\
132(74-140) \\
282(183-495) \\
113(98-166) \\
834(591-1570) \\
6131(2292-40,000) \\
151(38-172) \\
493(258-580) \\
24(11-41) \\
47(15-55)\end{array}$ & $\begin{array}{l}\text { ANA } \\
\text { Anti-ds DNA } \\
\text { Anti-U1RNP } \\
\text { Anti-Ro/SS-A } \\
\text { Anti-Sm } \\
\text { Antiphospholipid/LA } \\
\text { Direct Coombs Test + } \\
\text { C3 mg/dL (n.v 80-160) } \\
\text { C4 mg/dL (n.v 10-40) } \\
\text { Procalcitonin ng/mL (n.v. <0.5) }\end{array}$ & $\begin{array}{c}7(100 \%) \\
6(85 \%) \\
2(29 \%) \\
1(14 \%) \\
2(29 \%) \\
4(57 \%) \\
5(100 \%)^{\circ} \\
31(28-59) \\
10(4.8-17) \\
0.8(0.575-1.325)^{\#}\end{array}$ \\
\hline
\end{tabular}

*At least one of these criteria: malar rash; oral ulcers; photosensitivity; discoid lupus erythematosus; ${ }^{\circ}$ this test was available only for 5 patients; "this test was available only for 6 patients. CNS, central nervous system; WBC, white blood cells; HGB, haemoglobin; PLT, platelet; AST, aspartate aminotransferase; ALT, alanine aminotrasferase; $\mathrm{LDH}$, lactate dehydrogenase; ESR, erythrocyte sedimentation rate; CRP, C-reactive protein; ANA, antinuclear antibodies; anti-ds DNA, anti double stranded DNA antibodies; anti-RNP, anti-ribonucleoprotein antibodies; LA, Lupus anticoagulant: n.v., normal values.

cases required hospital admission, and 2 were admitted to the intensive care unit (ICU). In all cases MAS was simultaneous with the onset of SLE, and the main reasons for hospital admission were acute onset of fever and constitutional symptoms. At the moment of MAS, median SLEDAI value was 12 . Clinical manifestation of SLE were frequent (Table I). In particular, symptomatic heart involvement was present in 4 patients who developed pericarditis, in one case associated with myocarditis requiring left ventricular assist device implantation. Lung involvement was present in 4 cases with pleuritis; two patients developed acute severe respiratory failure due to acute respiratory distress syndrome. Kidney involvement was present in two patients with isolated proteinuria ( $>2$ grams $/ 24 \mathrm{~h}$ ), in one case with acute renal failure requiring dialysis. None of the patients underwent a renal biopsy. All patients had cytopenia in at least two cell lines (and 43\% of them of three lines). In all patients raised cytolysis enzymes, and in five (71\%) hyperferritine- mia, were observed. Antinuclear antibodies were detected in all patients, with very high titers of anti-dsDNA antibodies (median 720 IU; (IQR 91-744) nv <7). Median HLH score was 248 (IQR 226-263), with a median probability of having MAS of $99 \%$ (Table II). In all patients the Hscore was higher than 169, the cut-off for classification of reactive MAS in the original study (13); however, in one patient the score was lower than 190.5, a cut-off suggested by other authors to better perform in the classification of reactive MAS in patients with rheumatic diseases (17). In three cases bone marrow aspirate was not performed; nevertheless, the $\mathrm{H}$-score was higher than the suggested cut-offs in all these cases (Table II).

Workup for infections (including active viral replications) and malignancies was negative in all cases, except for one patient with positivity both for cytomegalovirus and herpes simplex 1 DNA, but without clinical manifestations. A mildly elevated serum procalcitonin level was found in 4 of 6 evaluable patients. All patients were 
treated with corticosteroids (including intravenous (iv) pulses of methylprednisolone in all cases); in one case iv dexamethasone was also administered. Concomitant treatments were: cyclosporin $\mathrm{A}$ in 6 patients (it was administered after the courses of iv corticosteroid), high-dose iv $\mathrm{IgG}$ in 5, mycophenolate mofetil in 1 , etoposide in 1 , plasma exchange in 1 , granulocyte colony-stimulating factor in 1 . Two patients required hemotransfusion. No death from MAS was observed.

One patient died 44 months after MAS for pulmonary adenocarcinoma. During follow-up (median: 54 months; IQR 39-70) no relapses of MAS have been recorded. At the last observation 5 patients were still being treated with low dose steroids $(<7.5$ $\mathrm{mg} /$ day), one patient with higher doses of prednisone $(15 \mathrm{mg} /$ day) because of SLE flare (central nervous system involvement); concomitant treatments were: mycophenolate mofetil in three patients, cyclosporine $\mathrm{A}$ in one, and azathioprine in one.

\section{DISCUSSION AND CONCLUSIONS}

In this study we report data on 7 adult SLE patients who developed MAS, collected in a single Italian rheumatology center. The prevalence of MAS was $1.4 \%$ among all patients followed at our center, and was similar to that reported in the literature in adult SLE patients (3-6). In our experience, in all cases MAS was diagnosed in untreated SLE patients with active disease. Although occasional cases of MAS associated with infections have also been reported in SLE (4 out of 41 cases collected in a recent review of the literature) (5), in the large majority of cases, MAS is therefore linked with active lupus (4-8). Although in our experience, MAS and SLE were diagnosed simultaneously and this was also the most frequent scenario in some other previous series $(4,8)$, recent data indicate that MAS may arise in patients with long disease duration in up to $50 \%$ of the cases (7).

Table II - H-score in seven patients with MAS-associated SLE.

\begin{tabular}{|c|c|c|c|c|c|c|c|}
\hline Parameter (criteria for scoring) & Patient 1 & Patient 2 & Patient 3 & Patient 4 & Patient 5 & Patient 6 & Patient 7 \\
\hline $\begin{array}{l}\text { Known underlying immunosuppression } \\
0 \text { (no) or } 18 \text { (yes) }\end{array}$ & 0 & 0 & 0 & 0 & 0 & 0 & 0 \\
\hline $\begin{array}{l}\text { Temperature }\left({ }^{\circ} \mathrm{C}\right) \\
0(38.4), 33(38.4-39.4) \text {, or } 49(39.4)\end{array}$ & 49 & 33 & 49 & 33 & 33 & 33 & 33 \\
\hline $\begin{array}{l}\text { Organomegaly } \\
0 \text { (no), } 23 \text { (hepatomegaly or splenomegaly), } \\
\text { or } 38 \text { (hepatomegaly and splenomegaly) }\end{array}$ & 23 & 38 & 23 & 23 & 38 & 38 & 38 \\
\hline $\begin{array}{l}\text { No. of cytopenias }{ }^{\star} \\
0 \text { ( } 1 \text { lineage), } 24 \text { (2 lineages), or } 34 \text { (3 lineages) }\end{array}$ & 24 & 24 & 24 & 24 & 34 & 34 & 34 \\
\hline $\begin{array}{l}\text { Ferritin (ng/mL) } \\
0(2000), 35(2000-6000) \text {, or } 50 \text { (6000) }\end{array}$ & 0 & 50 & 0 & 35 & 50 & 50 & 50 \\
\hline $\begin{array}{l}\text { Triglyceride (mmoles/L) } \\
0(1.5), 44(1.5-4) \text {, or } 64 \text { (>4) }\end{array}$ & 64 & 64 & 44 & 44 & 64 & 64 & 44 \\
\hline $\begin{array}{l}\text { Fibrinogen }(\mathrm{gm} / \mathrm{L}) \\
0(>2.5) \text { or } 30(<2.5)\end{array}$ & 30 & 30 & 30 & 30 & 30 & 30 & 30 \\
\hline $\begin{array}{l}\text { Serum glutamic oxaloacetic transaminase (IU/L) } \\
0(<30) \text { or } 19(>30)\end{array}$ & 19 & 19 & 19 & 19 & 19 & 19 & 19 \\
\hline $\begin{array}{l}\text { Hemophagocytosis features } \\
\text { on bone marrow aspirate } \\
0 \text { (no) or } 35 \text { (yes) }\end{array}$ & NA & NA & 0 & 35 & NA & 35 & 0 \\
\hline Total score ${ }^{\circ}$ & 209 & 258 & 189 & 243 & 268 & 303 & 248 \\
\hline
\end{tabular}

*Defined as a haemoglobin level of $<9.2 \mathrm{gm} / \mathrm{dL}$ and/or a leukocyte count of $<5000 / \mathrm{mm}^{3}$ and/or a platelet count of $<110,000 / \mathrm{mm}^{3}$; ${ }^{\circ}$ the cut-off suggested for the classification of reactive MAS in the original study was 169 (11). Other authors suggested a higher cutoff (190.5), in patients with rheumatic diseases (15). 
Differential diagnosis of MAS from lupus flares or infections that can mimic it is crucial for prompt and appropriate treatment and prevention of morbidity and mortality. Histopathological findings including hemophagocytosis are neither sensitive nor specific for this purpose (6). Indeed, in our experience, in three cases bone marrow aspirate was not performed and was not necessary to diagnose MAS. Clinical and laboratory signs may overlap between SLE and MAS, however there are some clues that can help in the distinction between the two disorders: hematologic involvement in SLE is very common, but pancytopenia or involvement of 2 cell lines are more frequent in SLE-associated MAS. Increases of cytolysis enzymes and ferritin are less frequently found in SLE than in MAS. Fever may be a red flag for possible MAS, particularly if the temperature is persistently above $38^{\circ}$, in the absence of signs and symptoms of underlying infection. While pericarditis is a frequent feature of SLE, the presence of myocarditis may be indicative of a severe associated MAS (4).

Validated diagnostic criteria for MAS in adults secondary to autoimmune disease and in particular SLE are therefore urgently needed. The H-score was designed to calculate the individual risk of adult patients having reactive MAS (15); however it was developed and validated in a population including a very small number of patients with SLE (and other autoimmune diseases) (15). The performance of the $\mathrm{H}$-score in the classification of MAS associated with rheumatic diseases was recently evaluated in a series of 30 patients, including 6 cases of SLE (17). In this study, a cut-off value for the $\mathrm{H}$-Score different from the original study performed better. The authors suggested that further studies may be warranted to determine optimum cut-off values in different patient populations. Here we present the largest population of adult SLE-associated MAS evaluated by the $\mathrm{H}$-score so far. Our data seem to confirm the utility of the $\mathrm{H}$-score in this setting, even if it should always be considered that sensitivity may not be $100 \%(13,17)$. However, since in our study all the patients also fulfilled the HLH-2004 classification criteria (10), it was not possible to evaluate the sensitivity of the $\mathrm{H}$-score as compared with the HLH-2004 criterion, the main limitation of which is indeed the lack of sensitivity, especially at the beginning of the MAS. For this purpose, case-control studies, in which patients with SLE-associated MAS are compared with SLE patients with fever but no MAS, might be appropriate, but these data are still lacking in the literature.

The therapeutic strategy for SLE-associated MAS has not yet been established (6, 8 ). In our experience, the combination of corticosteroids with other immunosuppressive medications was beneficial. A survey of the literature indicated that this combination therapy exerted more favorable effects, as compared with corticosteroids alone $(\mathrm{p}<0.05)(6)$. There has been no clear conclusion on which immunosuppressant is preferable for MAS in SLE. Cyclosporin A was the most frequently used in our, as in other series (8), and is generally preferred to other drugs for the lack of bone marrow suppression. Moreover, our data suggest that in most SLE patients the full therapeutic regimen developed for primary HLH (HLH-2004, including etoposide, cyclosporine, and dexamethasone, and intrathecal therapy with methotrexate and corticosteroids in selected patients) may be not necessary.

In our experience, no patient died because of MAS, but a mortality of $9.8 \%$ among 61 cases of SLE-associated MAS, identified through a review of the literature, was described (6), clearly indicating that this may be a potentially fatal condition. A very high mortality $(42.5 \%)$ was reported in a series of 41 cases of MAS associated with autoimmune diseases in a study from 2 Italian rheumatology centers, but cases of SLE were not separately analyzed (9). Moreover, in this study, older age and higher levels of serum ferritin were associated with mortality, and it has been reported that SLE patients are younger, and with lower levels of ferritin as compared with other adult patients with autoimmune disease-associated MAS (6). In another study, dermatomyosi- 
tis was identified as a risk factor for mortality in this situation (6).

In conclusion, MAS should be considered in febrile, cytopenic SLE patients. Ferritin, triglyceride and liver cytolysis enzymes blood levels may help in the diagnosis. A high clinical suspicion for myocarditis is mandatory in these cases, since this complication may be associated with the most severe forms (4): echography may be used as a screening test, since findings of global hypokinesis are strongly suggestive, but the gold standard for diagnosis remains MRI. The H-score can help in the classification of these patients, but its better sensitivity in this context has not yet been proven. Prompt treatment is necessary, considering the life-threatening potential of this condition.

\section{REFERENCES}

1. Sen ES, Steward CG, Ramanan AV. Diagnosing haemophagocytic syndrome. Arch Dis Child. 2017; 102: 279-84.

2. Wong KF, Hui PK, Chan JK, et al. The acute lupus hemophagocytic syndrome. Ann Intern Med. 1991; 114: 387-90.

3. Dhote R, Simon J, Papo T, et al. Reactive hemophagocytic syndrome in adult systemic disease: report of twenty-six cases and literature review. Arthritis Rheum. 2003; 49: 633-9.

4. Lambotte $\mathrm{O}$, Khellaf M, Harmouche H, et al. Characteristics and long-term outcome of 15 episodes of systemic lupus erythematosusassociated hemophagocytic syndrome. Medicine (Baltimore). 2006; 85: 169-82.

5. Kim JM, Kwok SK, Ju JH, et al. Reactive hemophagocytic syndrome in adult Korean patients with systemic lupus erythematosus: a case-control study and literature review. J Rheumatol. 2012; 39: 86-93.

6. Kumakura S, Murakawa Y. Clinical characteristics and treatment outcomes of autoimmuneassociated hemophagocytic syndrome in adults. Arthritis Rheumatol. 2014; 66: 2297 307.

7. Gavand PE, Serio I, Arnaud L, et al. Clinical spectrum and therapeutic management of sys- temic lupus erythematosus-associated macrophage activation syndrome: A study of 103 episodes in 89 adult patients. Autoimmun Rev. 2017; 16: 743-9.

8. Liu AC, Yang Y, Li MT, et al. Macrophage activation syndrome in systemic lupus erythematosus: a multicenter, case-control study in China. Clin Rheumatol. 2017 [Epub ahead of print].

9. Ruscitti P, Cipriani P, Ciccia F, et al. Prognostic factors of macrophage activation syndrome, at the time of diagnosis, in adult patients affected by autoimmune disease: Analysis of 41 cases collected in 2 rheumatologic centers. Autoimmun Rev. 2017; 16: 16-21.

10. Henter JI, Horne A, Aricò M, et al. HLH-2004: diagnostic and therapeutic guidelines for hemophagocytic lymphohistiocytosis. Pediatr Blood Cancer. 2007; 48: 124-31.

11. Ravelli A, Minoia F, Davì S, et al. Classification criteria for macrophage activation syndrome complicating systemic juvenile idiopathic arthritis: a European League Against Rheumatism/American College of Rheumatology/Paediatric Rheumatology International Trials Organisation collaborative initiative. Ann Rheum Dis. 2016; 75: 481-9.

12. Parodi A, Davì $S$, Pringe $A B$, et al. Macrophage activation syndrome in juvenile systemic lupus erythematosus: a multinational multicenter study of thirty-eight patients. Arthritis Rheum. 2009; 60: 3388-99.

13. Fardet L, Galicier L, Lambotte O, et al. Development and validation of a score for the diagnosis of reactive hemophagocytic syndrome (HScore). Arthritis Rheumatol. 2014; 66: 2613-20.

14. Hochberg MC. Updating the American College of Rheumatology revised criteria for the classification of systemic lupus erythematosus. Arthritis Rheum. 1997; 40: 1725.

15. Petri M, Orbai AM, Alarcón GS, et al. Derivation and validation of the Systemic Lupus International Collaborating Clinics classification criteria for systemic lupus erythematosus. Arthritis Rheum. 2012; 64: 2677-86.

16. Gladman DD, Ibanez D, Urowitz MB. Systemic lupus erythematosus disease activity index 2000. J Rheumatol. 2002; 29: 288-91.

17. Batu ED, Erden A, Seyhoğlu E, et al. Assessment of the HScore for reactive haemophagocytic syndrome in patients with rheumatic diseases. Scand J Rheumatol. 2017; 46: 44-8. 